\title{
A Complete Physics-Based Channel Parameter Simulation for Wave Propagation in a Forest Environment
}

\author{
Kamal Sarabandi, Fellow, IEEE, and Il-Suek Koh, Student Member, IEEE
}

\begin{abstract}
At HF through UHF frequencies, wave propagation in a forest environment is mainly attributed to a lateral wave which propagates at the canopy-air interface. Due to the existence of tree trunks, significant multiple scattering also occurs which is the dominant source of field fluctuations. Basically, the current induced in the tree trunks by the source and the lateral wave reradiate and generate higher order lateral waves and direct scattered waves. Using a full-wave analysis based on the method of moments in conjunction with Monte Carlo simulations, the effect of multiple scattering among a very large number of tree trunks is studied. It is shown that only scatterers near the source and the observation points contribute to the field fluctuations significantly. This result drastically simplifies the numerical complexity of the problem. Keeping about 200 tree trunks in the vicinity of the transmitter dipole and the receiver point, a Monte Carlo simulation is used to evaluate the statistics of the spatial and spectral behavior of the field at the receiver. Using a wide-band simulation, the temporal behavior (impulse response) is also studied as is performance of antenna arrays and the effects of different spatial diversity combining schemes in such a multipath environment.
\end{abstract}

Index Terms-Channel characterization, time-domain response, wave propagation.

\section{INTRODUCTION}

A CCURATE prediction of radio wave propagation in a communications channel is essential in the development and design of an efficient and low-cost wireless system. High-fidelity models are necessary in order to treat the tradeoff between radiated power and signal processing by addressing such issues as coherency, field variations, multipath, and dispersive (path delay) effects. Current channel models are heuristic in nature and have limited applicability. A physics-based approach to channel characterization gives insight into the mechanisms of radio wave propagation and inherently provides highly accurate results. With this as a motivation, a physics-based modeling approach is being pursued at the University of Michigan for which advanced and efficient electromagnetic diffraction models are being developed.

Due to relatively high attenuation rates, direct wave propagation in a forest environment is not possible over large distances at high frequencies. In the HF-UHF range where both the transmitter and receiver are embedded in the foliage, radio signals can propagate over relatively large distances. This peculiar behavior

Manuscript received May 16, 2000; revised August 25, 2000. This work was supported by the U.S. Army Research Office under Contract DAAD19-99-1-0325 and by the National Science Foundation under Contract ECS-9979376.

The authors are with the Radiation Laboratory, Department of Electrical Engineering and Computer Science, The University of Michigan, Ann Arbor, MI 48109-2122 USA (e-mail: saraband@eecs.umich.edu).

Publisher Item Identifier S 0018-926X(01)01269-8. is explained by a certain type of surface waves known as the lateral wave [1].

Mathematically speaking, the lateral wave is the contribution of the branch cut from the integral of the spectral representation of a dipole field inside a half-space dielectric. This formulation provides an expression for the mean-field assuming the canopy-air interface is smooth. For predicting the mean field more accurately, in a recent study [2], the effect of canopy-air interface roughness on the propagation of lateral waves was studied and it was shown that the canopy-air interface roughness reduces the mean field. As mentioned earlier, path loss only partially characterizes the channel, and other scattering mechanisms must be accounted for to complete the model. At UHF and lower frequencies, the dimensions of leaves and branches are small compared to wavelength and do not cause significant scattering which is the source of signal fluctuations, multipath, and dispersion. The effect of tree trunks which are electrically large create a highly scattering environment.

In this paper, the effect of tree trunks is accounted for by computing theirinteraction with the source and the lateral waves. Anumerical solution based on the method of moments (MoM) in conjunction with Monte Carlo simulation is proposed to evaluate the scattering effects of tree trunks. However, considering the number of tree trunks between the transmitter and receiver, it is quite obvious that a brute-force application of MoM is not possible due to the exorbitant memory and computation time requirements. To make the solution tractable while maintaining the model fidelity, three techniques are proposed: 1) simplification of the MoM formulation noting that tree trunks are sparsely distributed;2) simplification base on physical insight by noting that the scattered fields from tree trunks between the transmitter and receiver, but distant from them, are almostin-phase; and 3) simplification of field computation using the reciprocity theorem. In what follows, the forest model is described first and then the simplified MoM formulation is presented. This model is used to demonstrate that only scatterers near the source and observation points significantly contribute to the field fluctuations. Next, the near-field interaction of tree trunks with the source field and lateral waves are computed by application of reciprocity and using the MoM formulation. Finally, numerical simulations are presented, where the spatial decorrelations in a forest environment are examined and the performance of the antenna arrays and spatial diversity schemes evaluated.

\section{FOREST MODEL}

The model presented in this section is suitable for predicting the statistics of the field radiated by an elementary antenna em- 


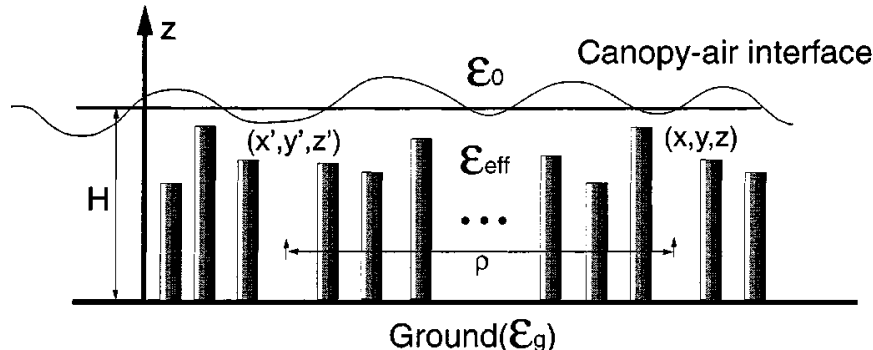

Fig. 1. Geometry of a forest model for characterization of wave propagation in a forest environment.

bedded in a forest environment and is valid for frequencies up to UHF. As mentioned earlier, since the dimensions of leaves and branches are small compared to the wavelength, the forest canopy can be modeled by a homogeneous dielectric . However, the trunks whose dimensions are comparable to or larger than a wavelength must be treated separately. Fig. 1 shows the geometry of the wave propagation problem, where both the source and observation points are within a dielectric slab with effective permittivity $\varepsilon_{\text {eff. }}$. This dielectric slab is assumed to have a smooth lower interface with a dielectric half-space (representing the ground) and a rough upper interface with air. Tree trunks are modeled by dielectric cylinders perpendicular to the ground plane having a relatively large height-to-diameter ratio. Ignoring the scattering from tree trunks, the mean field at the receiver is composed of the following components as depicted, in Fig. 2.

1) Geometric optics terms which include the direct propagation between the transmitter and receiver and reflected fields from the upper and lower interfaces. The mean reflected field from the upper interface is reduced exponentially where the exponent is proportional to the rms height of the canopy-air interface roughness [3]. These terms are only important when the receiver is relatively close to the transmitter, otherwise due to the lossy nature of the effective dielectric constant of the foliage these components do not contribute much. Basically the geometric optics terms exponentially decay with distance between the transmitter and the receiver.

2) Lateral wave (a diffraction term) which propagates along the canopy-air interface and decays proportionally to the reciprocal of the radial distance squared $\left(\sim 1 / \rho^{2}\right)$. The path loss associated with the lateral wave propagation increases with increasing foliage density (effective dielectric constant) and decreases by bringing the source or observation points closer to the canopy-air interface. The path loss increases as the canopy-air interface roughness (rms height) relative to wavelength increases. Close examination of the expression representing the lateral wave contribution reveals that the contribution can be attributed to a ray that emanates from the source along the critical angle, propagates along the canopy-air interface, and arrives at the receiver along the critical angle as shown in Fig. 2. Other higher order lateral wave contributions also exist, of which two are significant. One corresponds to the ray emitted from the source which reflects from the ground boundary, propagates along the critical angle and then travels along the interface $\left(E_{G L}\right)$. The other one is the reflected lateral wave from the ground which arrives at the receiver $\left(E_{L G}\right)$. These two contributions are also shown in Fig. 2

The expression for the electric field resulted from the asymptotic expansion of the spectral integral around the branch cut (Lateral wave) for a half-space dielectric with a smooth boundary is given by [2]

$$
\begin{aligned}
\overrightarrow{\mathbf{E}}_{L}= & \frac{j I l Z_{0}}{2 \pi(1-\kappa)^{1 / 4}} \\
& \cdot \frac{e^{-j k_{0} \rho} e^{-j k_{1} \sqrt{1-\kappa}\left(h+h^{\prime}\right)}}{\left[\left(h+h^{\prime}\right) \sqrt{\kappa}-\rho \sqrt{1-\kappa}\right]^{3 / 2} \rho^{1 / 2}} \overline{\overline{\mathbf{A}}} \cdot \hat{l}
\end{aligned}
$$

where $k_{1}=k_{0} \sqrt{\varepsilon_{\text {eff }}}, \rho$ is the radial distance between the transmitter and receiver, $h$ and $h^{\prime}$ are the depth of transmitter and receiver below the canopy-air interface, $I l$ is the current moment of the dipole, and $k_{0}$ and $Z_{0}$ are, respectively, the propagation constant and the characteristic impedance of free space. In (1), the unit vector $\hat{l}$ denotes the dipole orientation, $\kappa=1 / \varepsilon_{\text {eff }}$ where $\varepsilon_{\text {eff }}$ is the effective dielectric constant of foliage, and $\overline{\mathbf{A}}$ is a symmetric dyad given by

$$
\overline{\overline{\mathbf{A}}}=\left[\begin{array}{ccc}
\frac{\cos ^{2} \phi-\kappa}{\kappa} & \frac{\cos \phi \sin \phi}{\kappa} & \sqrt{\frac{1-\kappa}{\kappa}} \cos \phi \\
\frac{\cos \phi \sin \phi}{\kappa} & \frac{\sin ^{2} \phi-\kappa}{\kappa} & \sqrt{\frac{1-\kappa}{\kappa}} \sin \phi \\
\sqrt{\frac{1-\kappa}{\kappa}} \cos \phi & \sqrt{\frac{1-\kappa}{\kappa}} \sin \phi & 1
\end{array}\right] .
$$

For a canopy-air rough interface, $\overline{\overline{\mathbf{A}}}$ becomes more complex and can be found in [2]. Here $\phi$ is a cylindrical coordinate angle representing the location of observation point with respect to the transmitter. Assuming the canopy height is $H$, the lateral wave from the image of the source in the ground plane $\left(E_{G L}\right)$ is given by

$$
\begin{aligned}
\overrightarrow{\mathbf{E}}_{G L}= & \frac{j I l Z_{0}}{2 \pi(1-\kappa)^{1 / 4}} \\
& \cdot \frac{e^{-j k_{0} \rho} e^{-j k_{1} \sqrt{1-\kappa}\left(2 H-h+h^{\prime}\right)}}{\left[\left(2 H-h+h^{\prime}\right) \sqrt{\kappa}-\rho \sqrt{1-\kappa}\right]^{3 / 2} \rho^{1 / 2}} \overline{\overline{\mathbf{A}}} \cdot \overline{\overline{\mathbf{R}}} \cdot \hat{l}
\end{aligned}
$$

where $\overline{\overline{\mathbf{R}}}$ is the reflectivity matrix given by

$$
\overline{\overline{\mathbf{R}}}=\left[\begin{array}{ccc}
\sin ^{2} \phi R_{\perp}-\cos ^{2} \phi R_{\|} & -\sin \phi \cos \phi\left(R_{\perp}+R_{\|}\right) & 0 \\
-\sin \phi \cos \phi\left(R_{\perp}+R_{\|}\right) & \cos ^{2} \phi R_{\perp}-\sin ^{2} \phi R_{\|} & 0 \\
0 & 0 & R_{\|}
\end{array}\right]
$$

and $R_{\perp}$ and $R_{\|}$are the Fresnel reflection coefficients of the ground evaluated at the critical angle $\theta_{c}=\sin ^{-1} \sqrt{\kappa}$. Similarly $E_{L G}$ is given by

$$
\begin{aligned}
\overrightarrow{\mathbf{E}}_{L G}= & \frac{j I l Z_{0}}{2 \pi(1-\kappa)^{1 / 4}} \\
& \cdot \frac{e^{-j k_{0} \rho} e^{-j k_{1} \sqrt{1-\kappa}\left(2 H+h-h^{\prime}\right)}}{\left[\left(2 H+h-h^{\prime}\right) \sqrt{\kappa}-\rho \sqrt{1-\kappa}\right]^{3 / 2} \rho^{1 / 2}} \overline{\overline{\mathbf{R}}} \cdot \overline{\overline{\mathbf{A}}} \cdot \hat{l} .
\end{aligned}
$$




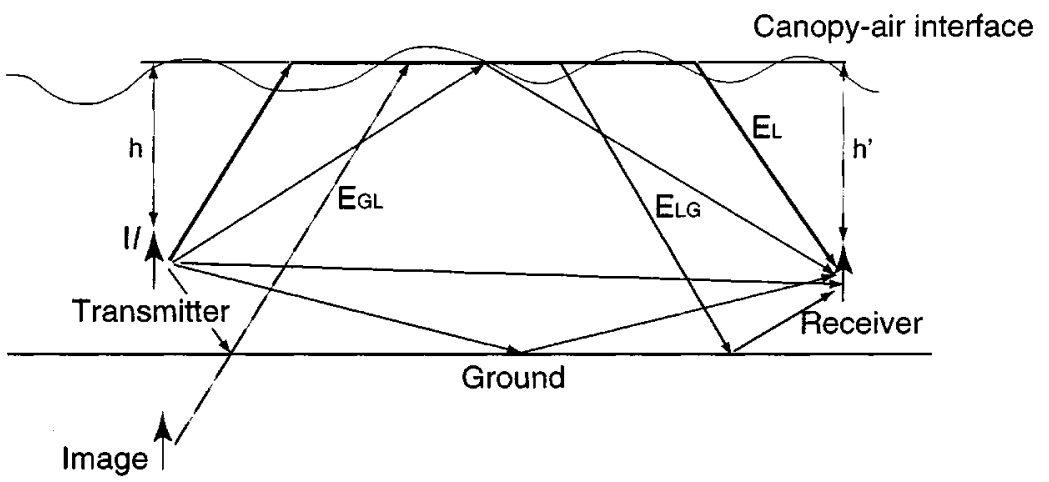

Fig. 2. Wave propagation mechanisms contributing to the mean field without tree trunks in a forest environment.

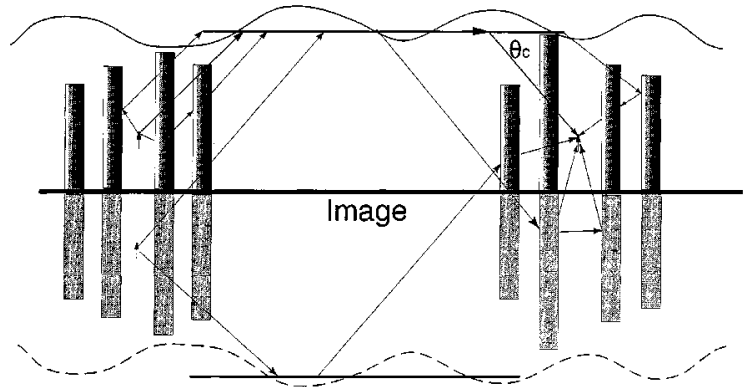

Fig. 3. Scattering mechanisms resulted from wave interaction with tree trunks in a forest environment.

In (3) and (5), the effects of the ground surface wave is ignored. The phase term of (1) indicates that the lateral wave is locally a plane wave propagating along the direction $\vec{k}_{1}=$ $k_{0}\left[\cos \phi \hat{x}+\sin \phi \hat{y}-\sqrt{\frac{1-\kappa}{\kappa}} \hat{z}\right]$ and also noting $\overrightarrow{\mathbf{E}}_{L} \cdot \vec{k}_{1}=$ 0 . The expression for the lateral wave contribution for rough canopy-air interface is more complicated than (1), but the mean field still represents a plane wave locally [2]. To include the effects of scattering from tree trunks, consider the geometry of the problem as shown in Fig. 3. The source and its image excite polarization currents in the dielectric cylinders which in turn reradiate and produce multiple scattering among the tree trunks and secondary lateral waves (lateral waves generated by scattering from the tree trunks) that arrive at the receiver. The sum of all lateral and secondary lateral waves will be referred to as the total incident wave in the vicinity of all receivers. The total incident wave excites polarization currents within the dielectric cylinders (tree trunks) near the receiver, which reradiate and together with the total incident fields constitute the total field at the receiver. A formal solution to the problem can be obtained rather easily by casting the formulation in terms of an integral equation for the polarization currents induced inside the dielectric cylinders. However, the brute-force solution of the integral equation using the method of moments is not tractable because of the large number of unknowns and the complex nature of the dyadic Green's function of the problem. To make the problem tractable, an accurate approximate solution is sought. An accurate approximate solution, considering the physics of the problem, can be obtained, as will be shown in the following section.

\section{REDUCED PROBLEM}

As mentioned before, estimation of field fluctuations in a forest environment requires the computation of multiple scattering effects, as well as the interaction of lateral waves with large number of dielectric cylinders. It is expected that the induced polarization currents in cylinders near the source be much stronger than those in cylinders distant from the source. Also, the contribution to the received fields from cylinders in the vicinity of the receiver is expected to be high. Although the contribution to the scattered fields from individual cylinders between the transmitter and receiver which are not in the close proximity of the either is relatively small, one may argue that there are many such cylinders and the overall contribution may be significant. Considering the path length between the transmitter to a cylinder and from the cylinder to the receiver, and noting that the scattering is strongest in the near-forward direction, the scattered fields from these cylinders arrive almost in-phase. Therefore, the scattered field from cylinders that are not close to the receiver and transmitter are not expected to contribute to the field fluctuations significantly. The effect of these scatterers may be accounted for by replacing them with an effective dielectric constant. To examine the validity of these postulations, a two-dimensional (2-D) medium consisting of infinite cylinders, excited by line source, is considered. Fig. 4(a) shows the geometry of the problem where a line source capable of supporting $z$-directed current (TM case) or $\rho$-directed current (TE case) is used as the transmitter. Fig. 4(b) shows the geometry of the reduced problem where the scatterers that are not close to the receiver and transmitter are replaced with an effective dielectric constant. Our objective here is to show that the mean and variance of the field in the original problem and the reduced problem are the same. The method of moments is used to solve these problems for a given arrangement of cylinders. Then, using Monte Carlo simulation, the desired field statistics are computed. In the implementation of the Monte Carlo simulation, the boundaries of the medium must be varied randomly by a few wavelengths to avoid coherence effects [4]. Position of cylinders are determined by a random number generator. In this filling process, the distance of a new cylinder is measured from the previous ones to ensure a minimum distance between the tree trunks. The filling process for each medium realization is continued until a desired number density is reached.

\section{A. The Method of Moments for M-Body Sparse Scatterers}

In this section, a numerical technique based on the method of moments appropriate for a relatively large number of sparsely distributed dielectric cylinders, illuminated at oblique incidence 


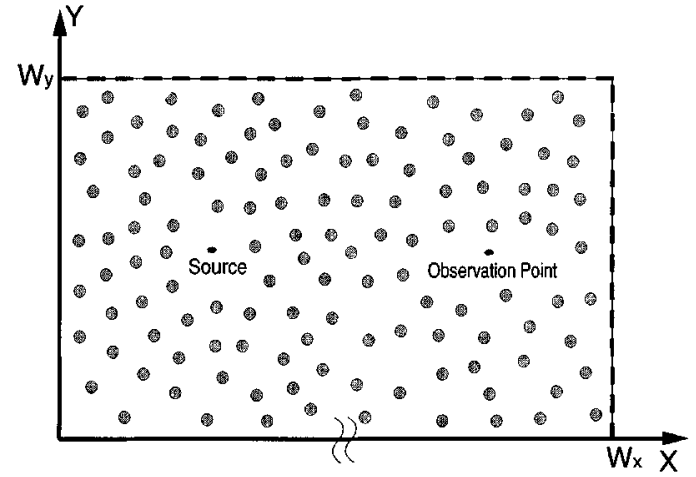

(a)

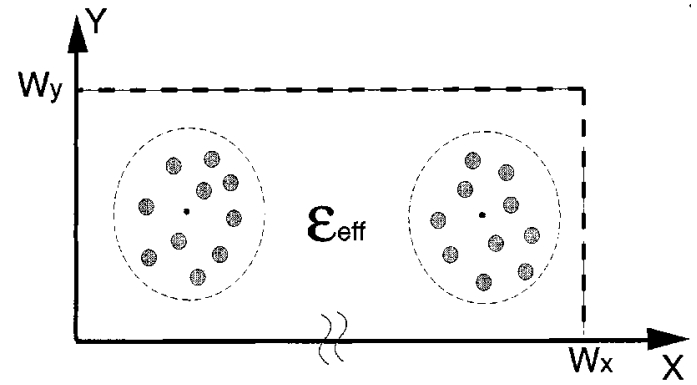

(b)

Fig. 4. A 2-D random medium consisting of (a) cylinders and (b) its statistically equivalent model. Also shown is a fictitious boundary used for the Monte Carlo simulation.

is described. The integral equation formulation for the induced polarization current for infinite cylinders whose axis are parallel to the $z$ axis is given by

$$
\begin{aligned}
& \frac{1}{\varepsilon_{r}-1} \overrightarrow{\mathbf{J}}(\vec{\rho}) \\
& \quad=-j k_{0} Y_{0} \overrightarrow{\mathbf{E}}^{i}+\sum_{n=1}^{N} \int_{s_{n}^{\prime}} \overline{\bar{G}}\left(\left|\vec{\rho}-\vec{\rho}^{\prime}\right|\right) \cdot \overrightarrow{\mathbf{J}}\left(\vec{\rho}^{\prime}\right) d s^{\prime}
\end{aligned}
$$

where

$\overrightarrow{\mathbf{E}}^{i} \quad$ represents the incident field having a propagation constant $k_{0}$,

$\varepsilon_{r} \quad$ is the relative dielectric constant of the cylinders, and

$s_{n}^{\prime} \quad$ is the cross section of the $n$th cylinder.

The explicit expression for the dyadic Green's function is given by

$$
\begin{aligned}
\overline{\bar{G}}\left(\left|\vec{\rho}-\vec{\rho}^{\prime}\right|\right) & \frac{j}{4}\left[\begin{array}{ccc}
k_{0}^{2}+\frac{\partial^{2}}{\partial x^{2}} & \frac{\partial^{2}}{\partial x \partial y} & -j k_{z} \frac{\partial}{\partial x} \\
\frac{\partial^{2}}{\partial x \partial y} & k_{0}^{2}+\frac{\partial^{2}}{\partial y^{2}} & -j k_{z} \frac{\partial}{\partial y} \\
-j k_{z} \frac{\partial}{\partial x} & -j k_{z} \frac{\partial}{\partial y} & k_{\rho}^{2}
\end{array}\right] \\
\cdot H_{0}^{(1)}\left(k_{\rho}\left|\vec{\rho}-\vec{\rho}^{\prime}\right|\right) &
\end{aligned}
$$

where $k_{z}$ is the propagation constant of the incident wave along the $z$ axis, and $k_{\rho}=\sqrt{k_{0}^{2}-k_{z}^{2}}$. Following the standard procedure of the MoM, the cross section of the cylinders are discretized into small cells over which the current distribution may

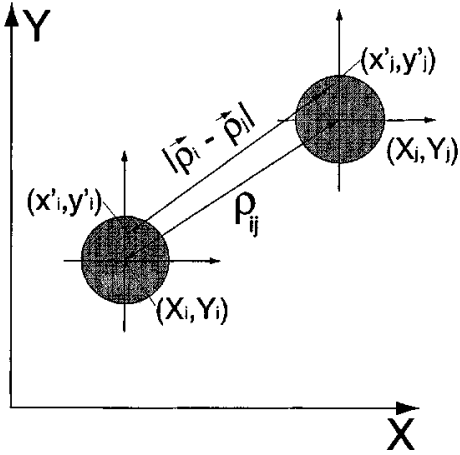

Fig. 5. Global and local coordinate systems used in the MoM formulation of sparse scatterers.

be considered a constant vector. Using point matching, the integral equation (6) can be cast in terms of a matrix equation of the form

$$
\overline{\bar{Z}} \cdot \overrightarrow{\mathbf{J}}=\overrightarrow{\mathbf{V}}
$$

where $\overline{\bar{Z}}$ is known as the impedance matrix. The size of the impedance matrix is proportional to the total number of cells for all $N$ cylinders and is a limiting factor with regard to computer memory. Noting that in a fully grown forest the tree trunks are sparsely distributed, storage of all elements of $\overline{\bar{Z}}$ can be avoided. In this case, the impedance matrix of the individual cylinders (block diagonal elements) are computed and stored. This can be limited to a few matrices corresponding a small number of cylinder, with different radii that represents the variability in tree trunk diameters. Next, the impedance matrix elements or the pairwise interaction between the cells at the center of the cylinders are computed and stored. The interaction between other elements are computed in an approximate manner as needed in the program and are not stored. For further clarification, consider the $i$ th and the $j$ th cylinders in the global coordinate system whose centers are respectively located at $\left(X_{i}, Y_{i}\right)$ and $\left(X_{j}, Y_{j}\right)$ as shown in Fig. 5. Suppose the interaction between two cells, whose local coordinate in the $i$ th and $j$ th system are, respectively, given by $\left(x_{i}^{\prime}, y_{i}^{\prime}\right)$ and $\left(x_{j}^{\prime}, y_{j}^{\prime}\right)$, are needed. In this case, using the Taylor series expansion,

$$
\begin{aligned}
& \left|\vec{\rho}_{i}-\vec{\rho}_{j}\right| \\
& \quad \approx \rho_{i j}+\frac{\left(X_{i}-X_{j}\right)\left(x_{i}^{\prime}-x_{j}^{\prime}\right)}{\rho_{i j}}+\frac{\left(Y_{i}-Y_{j}\right)\left(y_{i}^{\prime}-y_{j}^{\prime}\right)}{\rho_{i j}}
\end{aligned}
$$

where $\rho_{i j}=\sqrt{\left(X_{i}-X_{j}\right)^{2}+\left(Y_{i}-Y_{j}\right)^{2}}$ is the distance between the centers of the cylinders. The approximation in (9) can be used in the evaluation of integrals needed for the computation of matrix elements. For example,

$$
\begin{aligned}
I_{i^{\prime} j^{\prime}} & =\int_{x_{j}^{\prime}-\triangle / 2}^{x_{j}^{\prime}+\triangle / 2} \int_{y_{j}^{\prime}-\triangle / 2}^{y_{j}^{\prime}+\triangle / 2} H_{0}^{(1)}\left(k_{\rho}\left|\vec{\rho}_{i}-\vec{\rho}_{j}\right|\right) d x^{\prime} d y^{\prime} \\
& \approx \alpha_{n} H_{0}^{(1)}\left(k_{\rho} \rho_{i j}\right) \cdot e^{i \Phi_{i^{\prime} j^{\prime}}}=I_{i j} \cdot e^{i \Phi_{i^{\prime} j^{\prime}}}
\end{aligned}
$$

where $\Phi_{i^{\prime} j^{\prime}}=k_{\rho}\left[\left(X_{i}-X_{j}\right)\left(x_{i}^{\prime}-x_{j}^{\prime}\right)+\left(Y_{i}-Y_{j}\right)\left(y_{i}^{\prime}-y_{j}^{\prime}\right)\right] / \rho_{i j}$, and $\alpha_{n}=\triangle^{2}\left[1-\left(\left(k_{\rho} \triangle\right)^{2} / 24\right)\right]$. As mentioned earlier, $I_{i j}$ is stored and $I_{i^{\prime} j^{\prime}}$ is computed as needed. Using a sparse matrix storage scheme [5] and storing a small number of terms like 


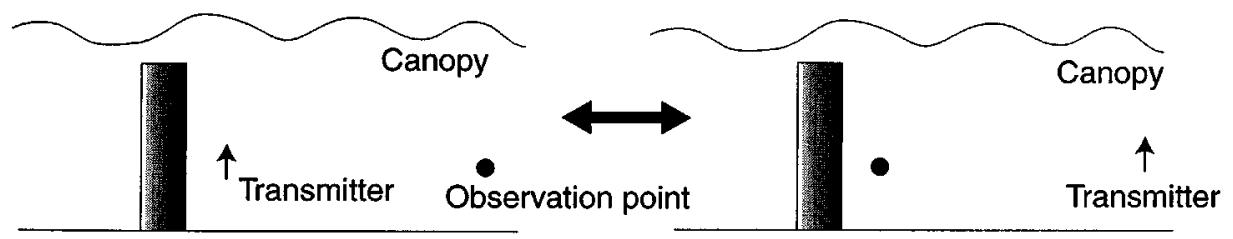

Fig. 6. Application of reciprocity theorem for the computation of scattered field of cylinder from a nearby dipole embedded in a dielectric slab above ground plane.

TABLE I

STORED MATRICES AND ARRAYS FOR THE IMPLEMENTATION OF M-BODY SPARSE SCATTERERS

\begin{tabular}{c|c}
\hline Stored Components & Size \\
\hline Imp. Matrix of individual cylinder & $M \times M:$ TM, $2 M \times 2 M: \overline{T E}$ \\
\hline$k_{\rho}\left(X_{i}-X_{j}\right) / \rho_{i j}, k_{\rho}\left(Y_{i}-Y_{j}\right) / \rho_{i j}$ & $\mathrm{M}(\mathrm{M}-1)$ array \\
\hline$\Phi_{i^{\prime} j^{\prime}}$ & $\mathrm{M}(\mathrm{M}-1)$ array \\
\hline $\cos ^{2} \theta_{i j}-\sin ^{2} \theta_{i j}$ & $\mathrm{M}(\mathrm{M}-1) / 2$ array \\
\hline
\end{tabular}

$\left(X_{i}-X_{j}\right) / \rho_{i j},\left(Y_{i}-Y_{j}\right) / \rho_{i j},\left(x_{i}^{\prime}-x_{j}^{\prime}\right)$, and $\left(y_{i}^{\prime}-y_{j}^{\prime}\right)$, the needed memory size is reduced. Similarly, for other terms of the dyadic Green's function that require spatial derivatives, we can use the following approximations:

$$
\begin{aligned}
\left(\frac{\partial^{2}}{\partial u^{2}}+k_{\rho}^{2}\right) I_{i^{\prime} j^{\prime}}= & \frac{\alpha_{n} k_{\rho}^{2}}{2}\left\{H_{0}^{(1)}\left(k_{\rho} \rho_{i j}\right) \pm H_{2}^{(1)}\left(k_{\rho} \rho_{i j}\right)\right. \\
& \left.\cdot\left[\cos ^{2} \theta_{i^{\prime} j^{\prime}}-\sin ^{2} \theta_{i^{\prime} j^{\prime}}\right]\right\} \cdot e^{\Phi_{i^{\prime} j^{\prime}}}
\end{aligned}
$$

where the positive and negative signs are used for $u=x$ for $u=y$, respectively. Also in (10)

$$
\begin{aligned}
& \cos ^{2} \theta_{i^{\prime} j^{\prime}}-\sin ^{2} \theta_{i^{\prime} j^{\prime}} \\
& \approx \frac{\cos ^{2} \theta_{i j}-\sin ^{2} \theta_{i j}+\left(a_{1}-a_{2}\right) / \rho_{i j}}{1+\left(a_{1}+a_{2}\right) / \rho_{i j}^{2}}, \\
& a_{1}=2\left(X_{i}-X_{j}\right)\left(x_{i}^{\prime}-x_{j}^{\prime}\right), \\
& a_{2}=2\left(Y_{i}-Y_{j}\right)\left(y_{i}^{\prime}-y_{j}^{\prime}\right), \\
& \theta_{i j}=\tan ^{-1}\left[\left(Y_{i}-Y_{j}\right) /\left(X_{i}-X_{i}\right)\right] .
\end{aligned}
$$

Moreover

$\frac{\partial I_{i^{\prime} j^{\prime}}}{\partial u} \approx \frac{\alpha_{n} k_{\rho}^{2}}{2}\left[H_{0}^{(1)}\left(k_{\rho} \rho_{i j}\right)+H_{2}^{(1)}\left(k_{\rho} \rho_{i j}\right)\right]\left(u_{i^{\prime}}-u_{j^{\prime}}\right) \cdot e^{\Phi_{i^{\prime} j^{\prime}}}$

for $u=x$ or $y$. Finally, for the $\partial^{2} I_{n} / \partial x \partial y$ term, we can use the equation shown at the bottom of the next page. Table I shows the submatrices and arrays that are stored in this scheme for $M$ identical cylinder, each discretized into $N$ pixels.

\section{B. Statistically Equivalent 2-D Medium}

In this section, a complete MoM solution for a large number of cylinders is used to verify the conjectures mentioned for the reduced problem. However, before presenting these results, the accuracy of the approximate MoM solution for the M-body, sparse scatterers problem is evaluated. An array of 50 equally spaced dielectric cylinders with $\varepsilon_{r}=5+j$ and radius $0.3 \mathrm{~m}$ are arranged along the $Y$ axis with a spacing of $2 \mathrm{~m}$. This array is illuminated by a $50-\mathrm{MHz}$ plane wave at an oblique incident angle $\theta=60^{\circ}$ and TE polarization. The scattered field computed by the exact MoM and the approximate MoM are compared along the curve, $y=x \ln x$. Small discrepancies are observed between the two solutions which are plotted Fig. 6 in as relative percentage error. Many other cases were also examined and it was found that, when the average ratio of cylinder spacing to cylinder radius is larger than 5, the approximate MoM is capable of producing very accurate results. Having confidence in the approximate MoM algorithm, simulation of wave propagation in a sparse random medium is carried out. A large number of dielectric cylinders (2000) confined in a rectangular box, as shown in Fig. 4, are considered. The box is chosen to have average dimensions of $50 \mathrm{~m} \times 800 \mathrm{~m}$. As mentioned earlier, to eliminate the coherence effect of finite box size (see [4]), the dimension of the box is varied by at least one wavelength in the Monte Carlo simulation. A line source is used as the transmitter, at location $(x=20, y=25)$ and the field is calculated at a point located at $(x=780, y=25)$. Many sample media are generated and the MoM solution for each sample is used to construct approximate statistics of the field at the receiving point. In specifics, the mean and standard deviation (SDV) of the field is monitored and the simulation is continued up until a convergence is reached. The contribution to the total mean field and standard deviation from scatterers in range bins $20 \mathrm{~m}$ wide are stored separately and are plotted in Fig. 8, for three frequencies of 10, 30, and $50 \mathrm{MHz}$. It is interesting to note that the contribution to the total standard deviation (field fluctuations) are mainly dominated by the scatterers near the source and observation points. This indicates that scatterers between, but not close to, the transmitter and receiver are not significant sources of field fluctuations and can be replaced with an effective dielectric constant. The geometry of the reduced problem is shown in Fig. 4(b) where 600 cylinders near the source and 250 cylinders near the observation point are kept and the rest are replaced with an effect dielectric medium. In this simulation, a cylinder density $0.05 / \mathrm{m}^{2}$ is used and the comparison of standard deviation for the complete and reduced problems for the TM case is shown in Fig. 9. As can be seen in Fig. 9, the reduced and complete problems produce effectively

$$
\frac{\partial^{2} I_{i^{\prime} j^{\prime}}}{\partial x \partial y}=\frac{\left(X_{i}-X_{j}\right)\left(Y_{i}-Y_{j}\right)+\left(X_{i}-X_{j}\right)\left(y_{i}^{\prime}-y_{i}^{\prime}\right)+\left(Y_{i}-Y_{j}\right)\left(x_{i}^{\prime}-x_{j}^{\prime}\right)}{\rho_{i j}^{2}+\left(a_{1}+a_{2}\right)} \cdot k_{\rho}^{2} H_{2}^{(1)}\left(k_{\rho} \rho_{i j}\right) \cdot e^{\Phi_{i^{\prime} j^{\prime}}} .
$$


the same field variations. To examine the field variance only, far less scatterers produce the same result. Fig. 10(a)-(c) show, respectively, the field variance for TE [(a) and (b)] and TM [(c)] excitation using 200 cylinders near the source and 150 cylinders near the receiver. For these simulations, cylinder density $0.01 / \mathrm{m}^{2}$ is used. For the reduced problem, the effective medium is anisotropic and its dielectric constant is a tensor given by [4]

$$
\begin{aligned}
& \varepsilon_{\mathrm{eff}}=\varepsilon_{h}+\left(\varepsilon_{i}-\varepsilon_{h}\right) f, \quad \text { for TM } \\
& \varepsilon_{\mathrm{eff}}=\varepsilon_{h}+f\left(\varepsilon_{i}-\varepsilon_{h}\right) \frac{2}{\varepsilon_{i}+\varepsilon_{h}}, \quad \text { for TE. }
\end{aligned}
$$

\section{FAST-FIELD CALCULATION BASED ON RECIPROCITY}

As our goal in this investigation is the computation of the fields of a dipole in a forest environment, computation of polarization currents in tree trunks when illuminated by the dipole is needed. Even with the simplification mentioned in the previous section, a three-dimensional (3-D) scattering problem which includes more than 200 cylinders is not tractable numerically. In this section, we demonstrate a procedure where, with the help of reciprocity theorem, the 3-D scattering problem is first reduced to an equivalent 2-D problem, which is then solved by the method of moments. To demonstrate this procedure, let us first consider a short dipole near a single cylinder, as shown in Fig. 7. The field at the receiver is the sum of the field of the dipole and the radiated field from the polarization current induced in the dielectric cylinder embedded in the canopy above the ground. Since the observation point is in the far field of the cylinder and the dipole, reciprocity can be applied to simplify the problem. According to the reciprocity theorem [6], the vertical component of the received field for a dipole excitation with orientation $\hat{l}$ is equal to the $\hat{l}$ component of the field near the cylinder of the same dipole oriented vertically and located at the observation point. According to (1), the field of the dipole (in the modified problem) illuminating the dielectric cylinder is locally plane wave. Also, noting that the induced polarization currents in a finite, long dielectric cylinder is approximately the same as those of an infinite cylinder having the same radius and dielectric constant [7], [8], the MoM solution for 2-D problems can be used to find the induced polarization currents. Once this polarization current is obtained, the near field can easily be computed and the expression for it given in the Appendix. The same procedure is applied to find the contribution of all cylinders in the vicinity of the transmitter, at the receiver. To compute the effect of the scattered field of cylinders near the receiver, the fields of the dipole and all cylinders in its vicinity are computed at each cylinder location near the receiver. Again, these fields are locally plane waves illuminating tree trunks near the receiver at an oblique incidence equal to the critical angle. MoM is used to find the induced polarization current in cylinders near the receiver from which the scattered field is computed. Hence, the contribution from all cylinders near the transmitter and receiver are included. To account for the effect of the forest floor, the geometric optics images of the dipole and lateral waves on the ground plane are considered and their contributions are evaluated using a similar procedure. It is worth mentioning that, in this case, the number of cylinders which need to be kept in the vicinity of the transmitter and receiver for the reduced problem is expected to be

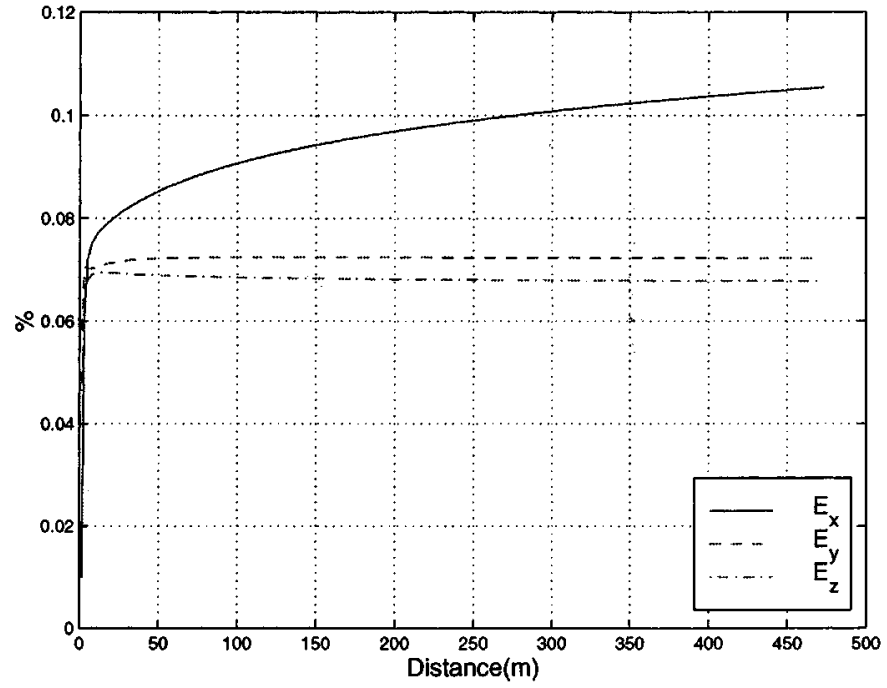

(a)



(b)

Fig. 7. Relative percentage error in (a) magnitude and (b) phase of scattered electric field from 50 infinite dielectric cylinders that are located along $y$-axis with $2 \mathrm{~m}$ separation when a TE plane wave is obliquely incident with $\theta=60^{\circ}$ at $50 \mathrm{MHz}$

smaller than what was obtained for the 2-D problem. Basically, for the dipole excitation where the finite tree trunks are illuminated by the resulting plane waves at oblique incidence, fewer number of cylinders can interact with each other.

\section{NUMERICAL SIMULATION}

Based on the rigorous electromagnetic model described in the previous section, a very accurate propagation model which provides a complete channel characterization of forest media is possible through Monte Carlo simulations. To examine the effect of tree trunks on the field of a transmitter in a forest, we first consider a single tree trunk in the near-field of a short dipole. For this example, a dielectric cylinder with permittivity $\varepsilon=5+j$, radius $a=35 \mathrm{~cm}$, and height $h_{c}=15 \mathrm{~m}$ is considered in a forest with a canopy having $\varepsilon_{\text {eff }}=1.03+j 0.036$ and height $H=20 \mathrm{~m}$. A vertical dipole transmitting at $90 \mathrm{MHz}$ is placed at a distance of $10 \mathrm{~cm}$ from the surface of the cylinder and is moved up and down, 


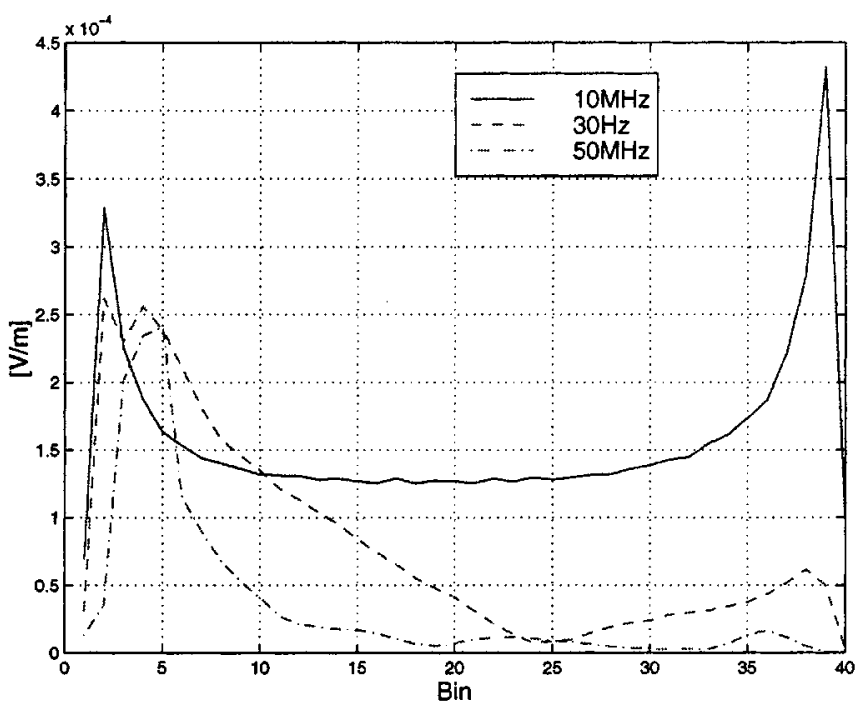

(a)

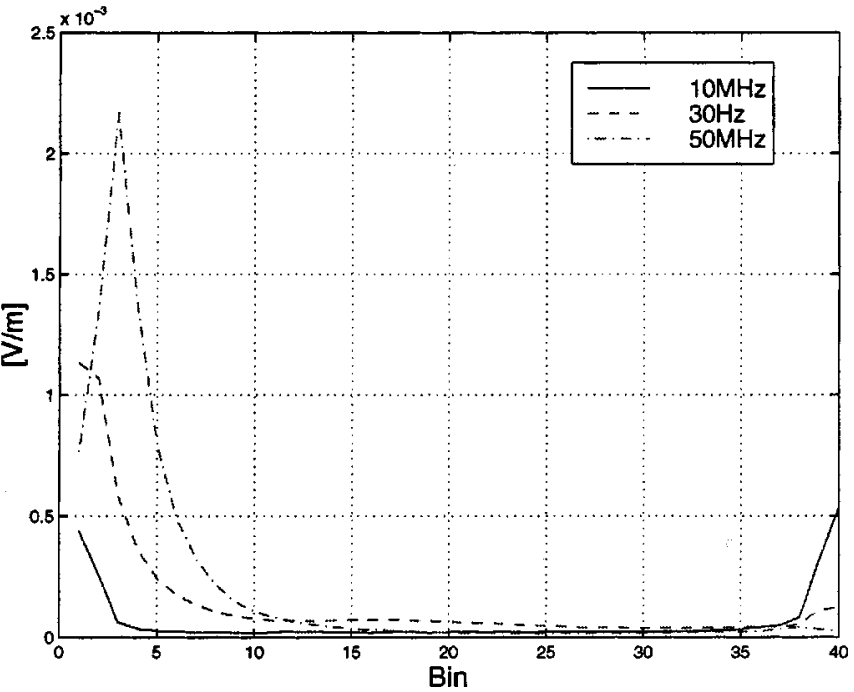

(b)

Fig. 8. Mean and standard deviation of scattered electric field generated by scatterers in each 20-m bin with TM source $(x=20, y=25)$ and an observation point $(x=780, y=25)$. Three different frequencies are used, 10,30, and $50 \mathrm{MHz}$ and total number of scatterers is 2000. (a) Magnitude of mean. (b) Magnitude of standard deviation.

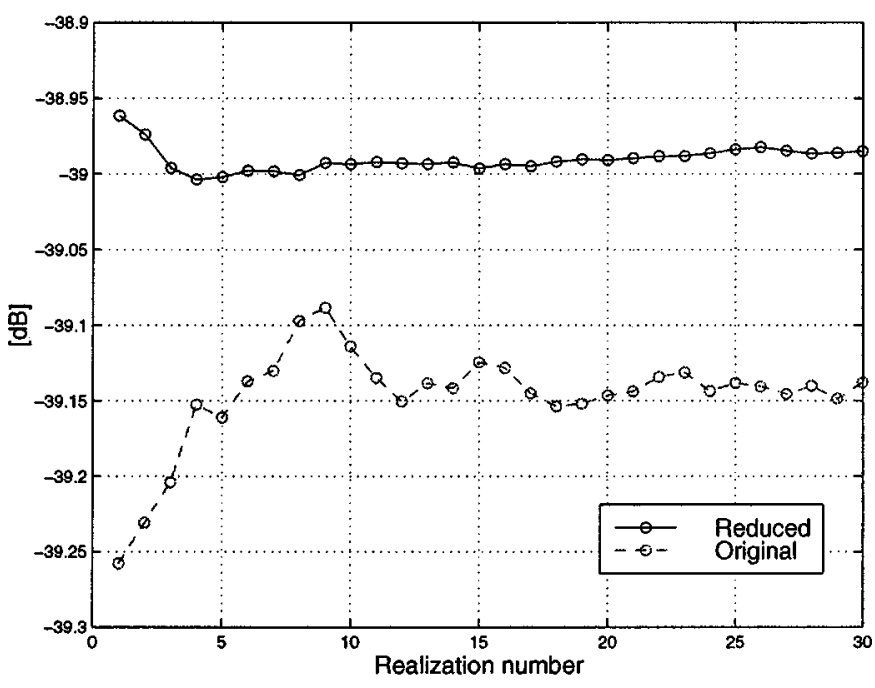

(a)

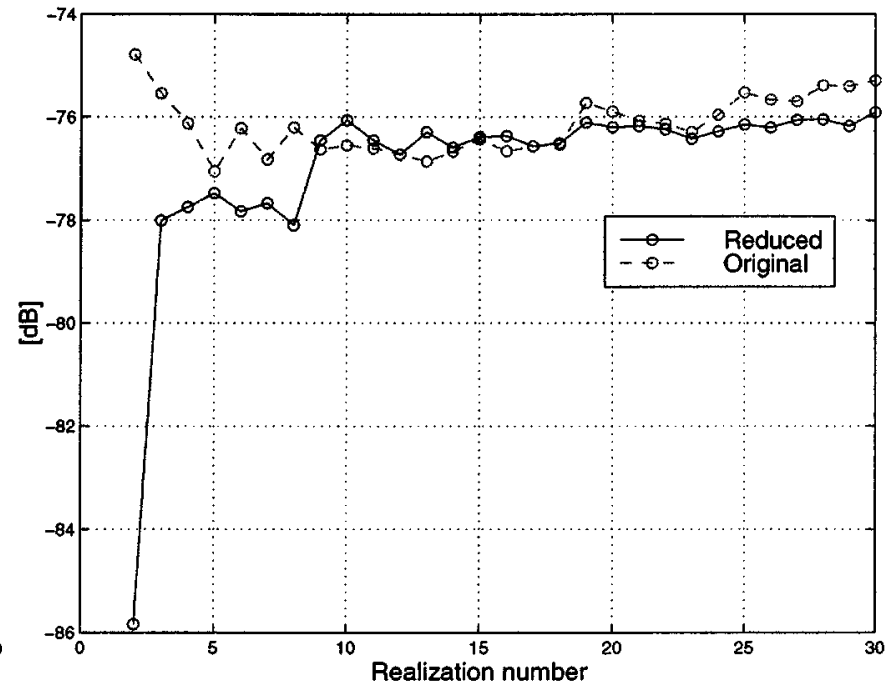

(b)

Fig. 9. Comparison of (a) the mean field and (b) standard deviation of $E_{z}$ of the reduced problem that keeps 600 scatterers near the source and 250 scatterers near the observation point with those of the complete problem that contains 2000 scatterers, with TM line source excitation.

and around the cylinder and its field is computed at an observation point $1 \mathrm{~km}$ away from the cylinder and $5 \mathrm{~m}$ above the ground plane. Fig. 11 shows the normalized $z$ component of the field at the receiver as a function of dipole height above the ground and for three azimuthal angles around the cylinder. The normalization here is with respect to the field of the dipole in the absence of the cylinder. It is shown that the field at the receiving point fluctuate as the dipole is moved along the cylinder axis. This fluctuation is a result of constructive and destructive interference of the direct field, scattered field from the cylinder, and their images on the ground plane. Since the cylinder radius is small compared to the wavelength, a gentle variation is observed as the dipole is moved around the cylinder. It is interesting to note that, for most dipole locations, existence of the cylinder in the near-field region of the dipole enhances the field at the receiver since the tree trunk acts as a passive radiator. Next, computation of path loss and field standard deviation is considered for the forest of the previous example. In this case, tree trunks having an average height $15 \mathrm{~m}$ and height standard deviation of $1 \mathrm{~m}$ with number density $0.05 / \mathrm{m}^{2}$ and dielectric constant of $\varepsilon=5+j$ are also included. As mentioned before, the number of cylinders we need to keep for the reduced problem is expected to be smaller than those for the 2-D problem. Here we kept 200 cylinders near the transmitter located at the origin $3 \mathrm{~m}$ above the ground and 50 cylinders near the receiver located $1 \mathrm{~km}$ away from the transmitter and 5 $\mathrm{m}$ above the ground. Table II shows the path loss (with respect to the free space) of the normal component of the wave and its standard deviation at $50 \mathrm{MHz}$ for a vertical dipole. Also shown 


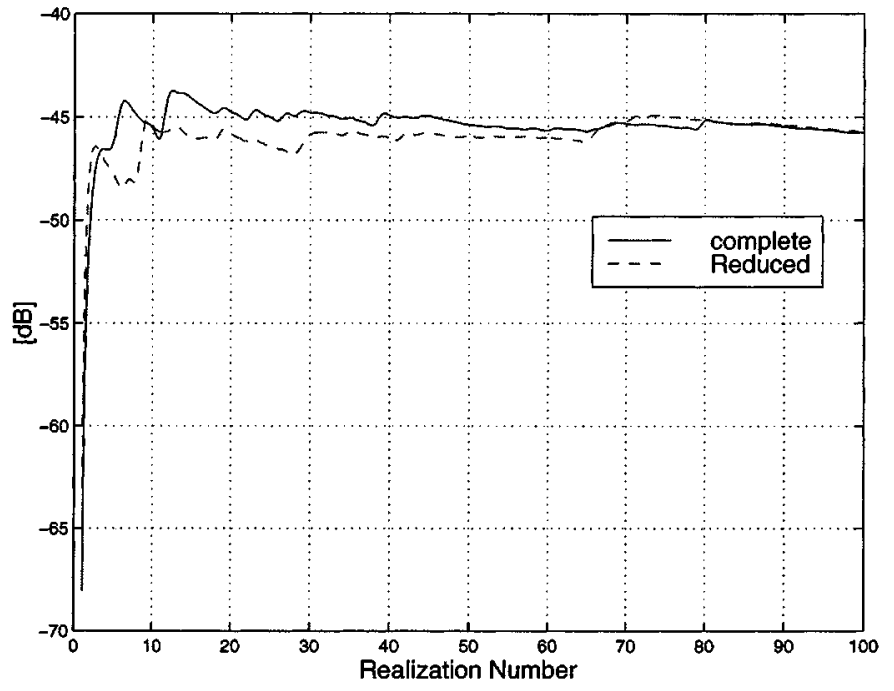

(a)

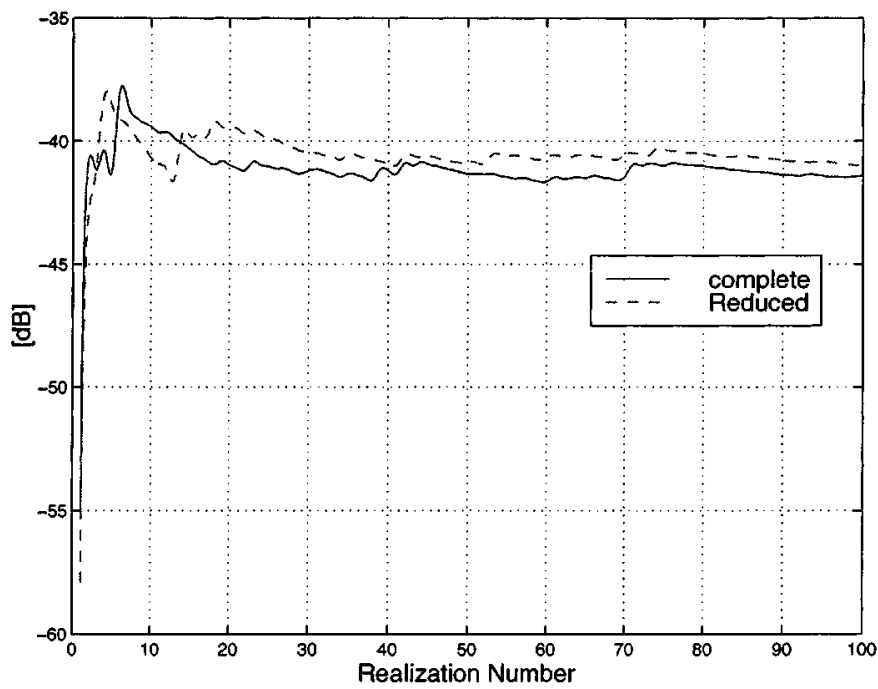

(b)

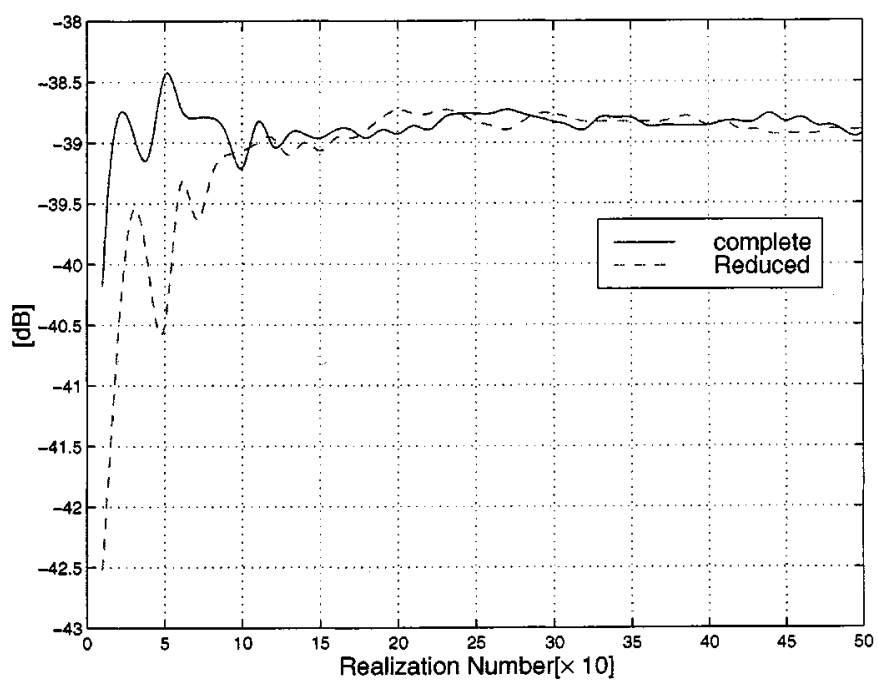

(c)

Fig. 10. Comparison of the standard deviation for (a) and (b) TE and (c) TM excitations of the complete and reduced problems that keeps 200 (TE) or 150 (TM) scatterers near the source and 150 scatterers near the observation point with those of the complete problem that contains 500 scatterers, with TE line source excitation. in Table II are the path loss and standard deviation if 390 cylinders (250 near the source and 140 near the receiver) are kept. The Monte Carlo simulation converges after about 30 realizations and is shown that the results based on 200 cylinders is very close to those based on 390 cylinders. This convergence test indicates that a moderate number of scatterers (about 200) is sufficient for accurate characterization of field variance. The results also indicate a standard-to-mean ratio deviation of almost unity for the random variable $E_{z}$. Using different forest parameters and frequencies (at $\mathrm{HF}$ band), random variable $E_{z}$ showed a similar behavior. That is, the statistics of the channel follows Ricean statistics with a standard deviation-to-mean ratio ranging from 0.5 to 2 . This is different than Rayleigh statistics which is commonly assumed for communication channels when there is no line-of-sight propagation path. Significant nonzero mean-field in this case is a direct result of contributions from the lateral wave.

For communication channels with significant multipath, antenna arrays are usually used to mitigate fading. In this case, because of the existence of a considerable coherent mean field, antenna arrays may provide coherent gain. To investigate the performance of antenna arrays in a forest environment two basic configurations, namely, broadside and end-fire are considered. For the broadside configuration, four vertical dipoles are arranged along a line perpendicular to the line between the transmitter and receiver points with a spacing of $\lambda / 2$. The four elements of the end-fire array are placed along the line between the transmitter and receiver separated by $7 \lambda / 16$ and having a progressive phase factor of $-7 \pi / 8$ [9]. The field of these two arrays are evaluated and the path loss and SDV-to-mean ratio is reported in Table II. It is found that the broadside array has about $11 \mathrm{~dB}$ less path loss than the end-fire array. This is very close to the 12-dB gain of a 4-element array and indicates that the field of all four elements of the broadside array arrive at the receivers almost coherently whereas those of the end-fire array are incoherent. This behavior can be understood by considering the spatial correlation function in the forest environment. The spatial correlation coefficient between two points $\vec{r}_{1}$ and $\vec{r}_{2}$ is defined as [11]

$$
C_{s}\left(\vec{r}_{1}, \vec{r}_{2}\right)=\frac{\operatorname{Cov}\left[\vec{E}\left(\vec{r}_{1}\right), \vec{E}\left(\vec{r}_{2}\right)\right]}{\sigma_{E_{1}} \sigma_{E_{2}}}
$$

where $\sigma_{E_{1}}$ and $\sigma_{E_{2}}$ are the variance of the field at $\vec{r}_{1}$ and $\vec{r}_{2}$, respectively. A grid of nine points along the $x$-axis (direction between the transmitter and receiver) and nine points along the $y$-axis separated by $\lambda / 2$ that is $1 \mathrm{~km}$ from the transmitter is generated and components of the electric field are calculated many times for different cylinder arrangements in order to compute the correlation coefficient. Fig. 12(a) and (b) show the magnitude and phase of $C_{s}\left(\vec{r}_{1}, \vec{r}_{2}\right)$ along the $x$ and $y$ axis, respectively. It is shown that the signal along the $y$-axis remains coherent over much larger distance than along the $x$-axis. This result is in agreement with the observed behavior of broadside and end-fire antenna arrays. Sampling theorem [10]-[12] is used to generate the smooth curves that goes in between the discrete points in Fig. 12.

To study the path-delay effects, the impulse response of the channel must be characterized. The impulse response cannot 


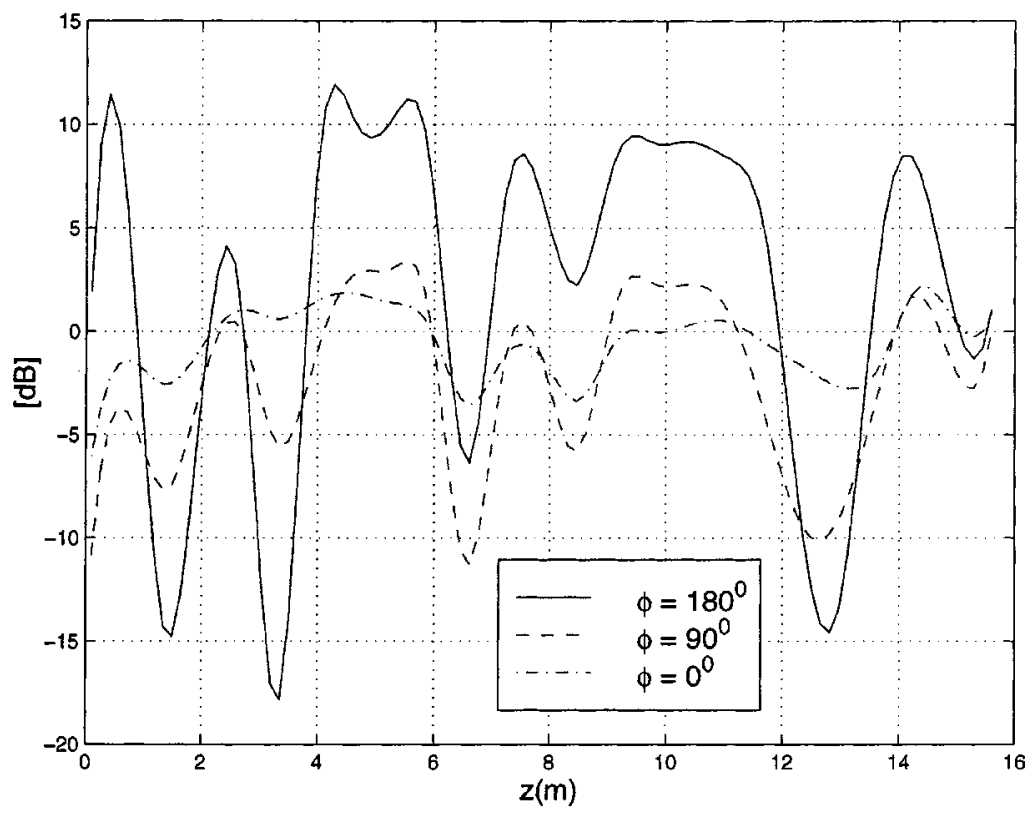

Fig. 11. The ratio of the fields of a dipole with and without a tree trunk as a function of dipole height $(z)$ and azimuthal angle around the cylinder. The dipole is vertical and $10 \mathrm{~cm}$ away from the cylinder surface and observation point is $1 \mathrm{~km}$ away.

TABLE II

Path-Loss and Standard DeViation-to-Mean Ratio of THe Field of a VERTICAL DIPOLE ANDFOUR -ELEMENT DIPOLE ARRAYS IN THE FOREST WITH $\varepsilon_{\mathrm{cff}}=1.03+j 0.036$, CANOPY HEIGHT $H=20 \mathrm{~m}$, TREE DENSITY $0.05 / \mathrm{m}^{2}$, AND TREE TRUNK HeIGHT OF $H=15 \mathrm{~m}$

\begin{tabular}{c|c|c|c|c}
\hline \multirow{2}{*}{ Antenna Configuration } & \multicolumn{2}{|c|}{ Path-loss(dB) } & \multicolumn{2}{c}{ SDV/Mean(dB) } \\
\cline { 2 - 5 } & 200 cyl. & $390 \mathrm{cyl}$. & $200 \mathrm{cyl}$ & $390 \mathrm{cyl}$ \\
\hline single dipole & 47.5 & 48.16 & 1.42 & 1.16 \\
\hline 4-element Broadside & 48 & & 3 & \\
\hline 4-element End-fire & 59 & & 2.45 & \\
\hline
\end{tabular}

be directly determined. However, by characterizing frequency response over a wide bandwidth, the impulse response can be determined by applying the Fourier transformation to generate time-domain (range) information. Direct application of FFT is not computationally efficient considering the number of frequency points which are needed in order to avoid aliasing. For example, with a receiver at a distance of $1.5 \mathrm{~km}$ from the transmitter a maximum frequency spacing of $200 \mathrm{KHz}$ is required. A pulsewidth of $12.5 \mathrm{~ns}$ corresponds to $80 \mathrm{-MHz}$ bandwidth which can be used to resolve path delays $3.75 \mathrm{~m}$ apart. In this case, for each forest realization, 400 frequency points must be simulated. To circumvent this difficulty, we used a nonuniform frequency sampling scheme. Gauss quadrature integration [12] is used to evaluate the Fourier transform. The order of Legendre polynomial should be chosen so that the minimum distance between the zeros of the Legendre polynomial is smaller than the minimum frequency spacing required to avoid aliasing ( $200 \mathrm{KHz}$ for the above example). Impulse response of the forest considered in the previous example is simulated at HF using a bandwidth of $10-90 \mathrm{MHz}$. A receiver at a distance of $1 \mathrm{~km}$ from the transmitter is considered. It is expected that the dispersion (pulse spreading) will be observed due to multiple scattering among tree trunks and the frequency-dependent path loss of lateral waves. Fig. 13 shows the frequency response of the received field in the absence of

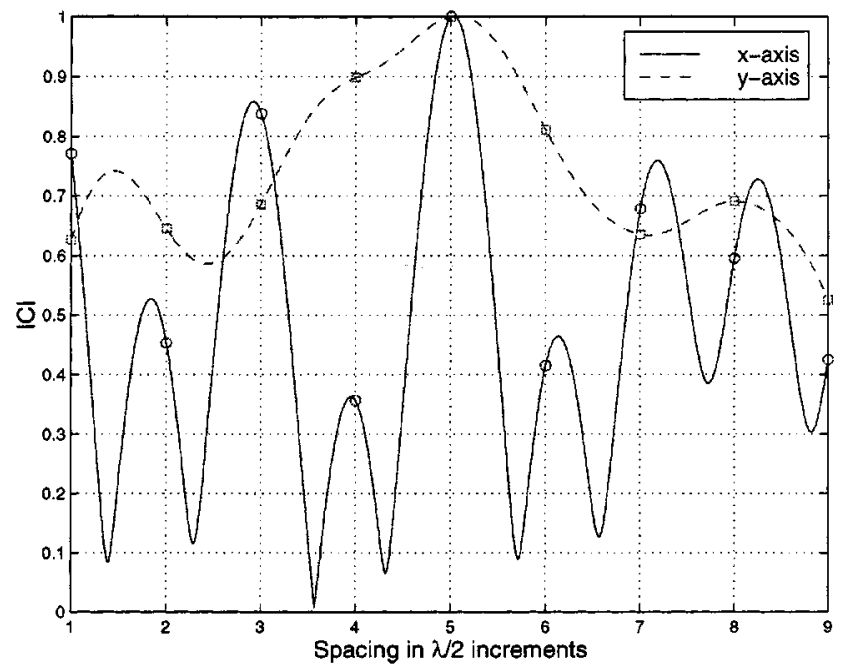

(a)

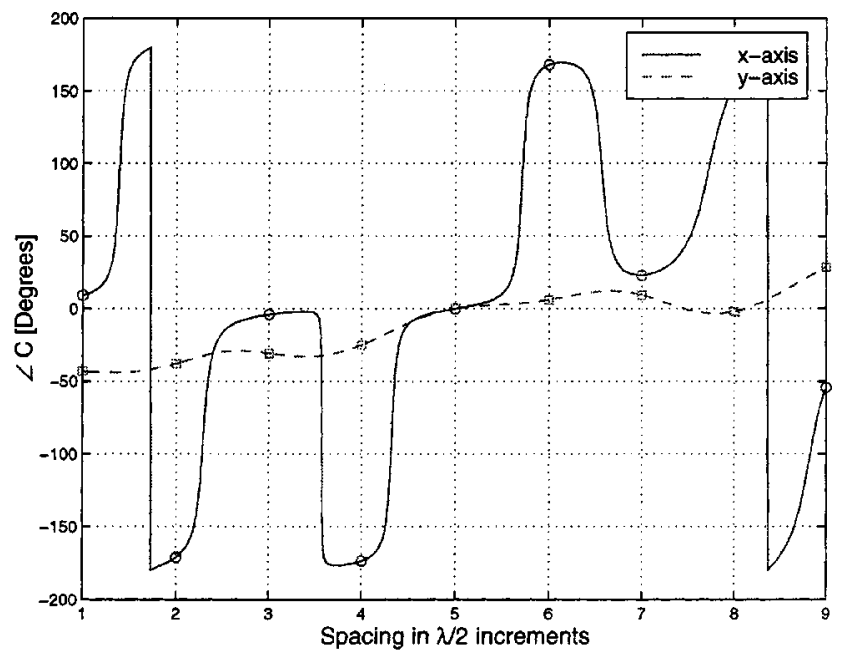

(b)

Fig. 12. Spatial correlation of (a) magnitude and (b) phase of field of a vertical dipole $\left(E_{z}\right)$ along the $x$ and $y$ axis. 


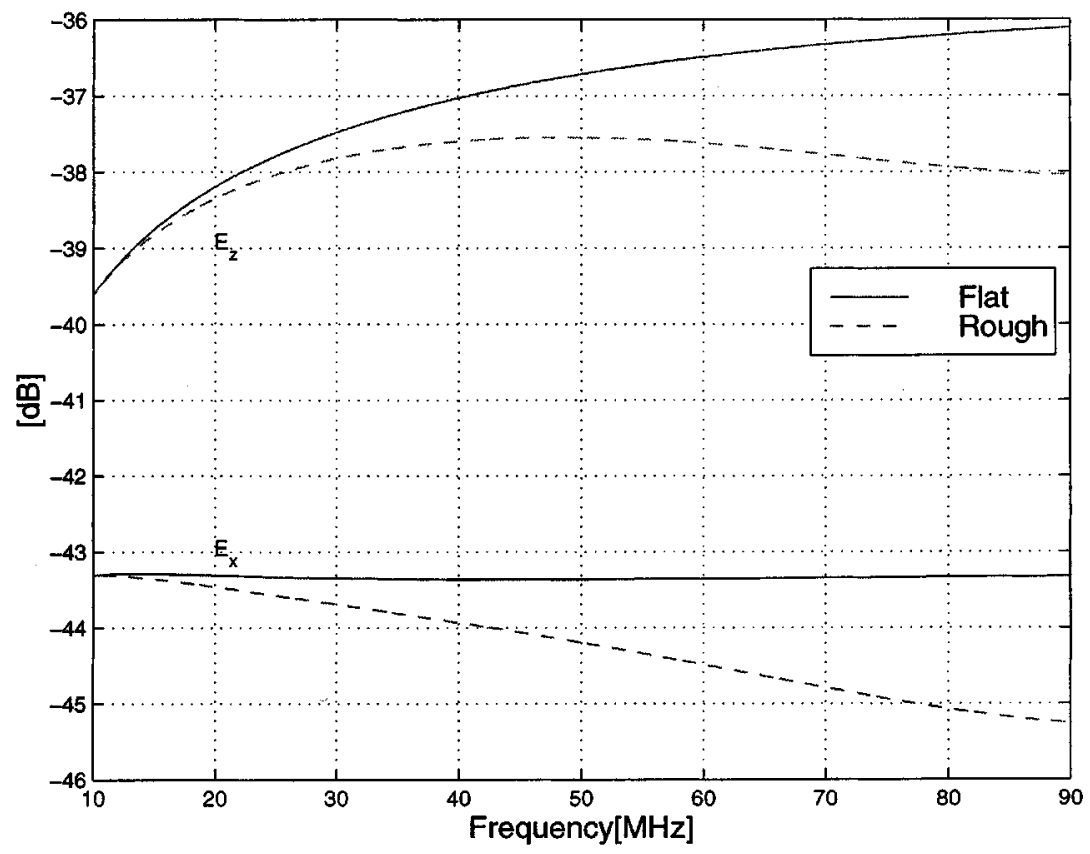

Fig. 13. Magnitude of mean field as function of frequency in the absence of tree trunks and the ground plane. RMS height is $2 \mathrm{~m}$ and $\varepsilon_{\text {cff }}=1.03+j 0.036$ and the transmitter and receiver are 17 and $15 \mathrm{~m}$ below the canopy-air interface, respectively.

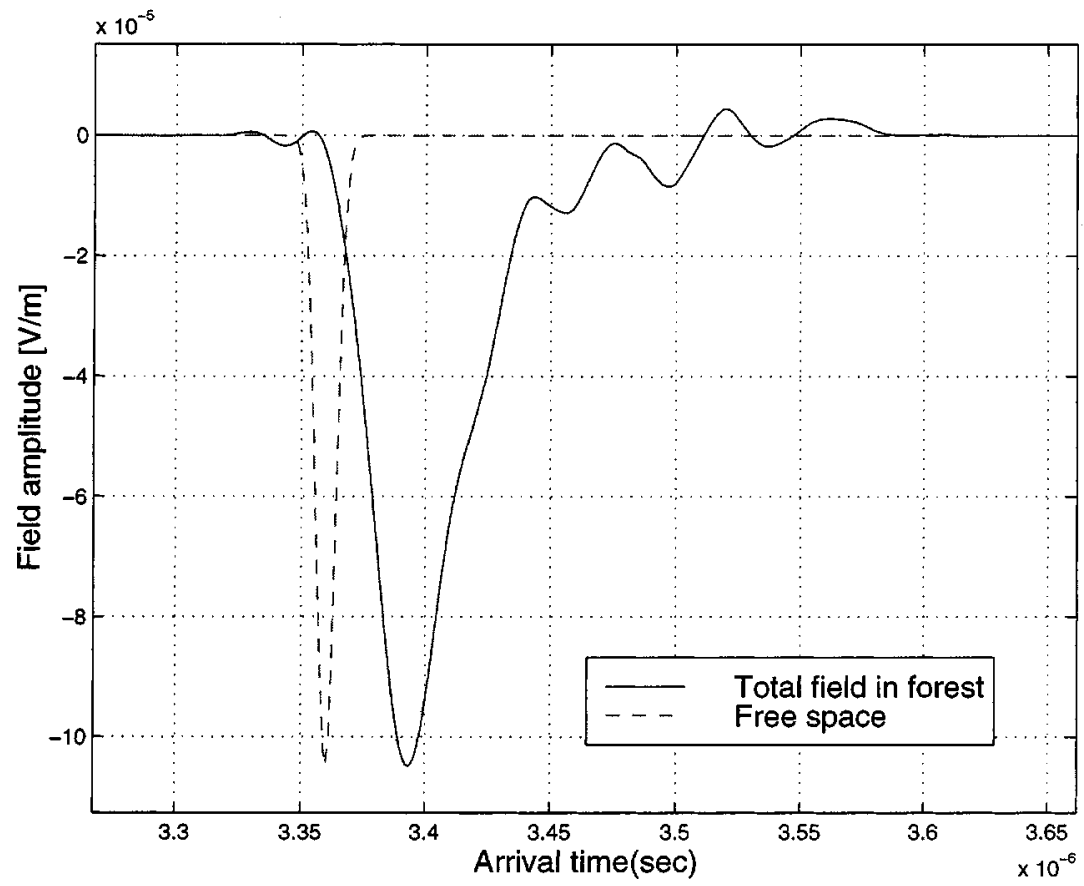

Fig. 14. Impulse response with Gaussian pulse excitation on the $\hat{z}$ directed dipole. Forty nonuniformly spaced sampling points are used. Dotted line is free-space response multiplied by the path loss evaluated at $50 \mathrm{MHz}$ (see Table II).

the tree trunks and the ground plane for two cases: 1) smooth canopy-air interface and 2) rough canopy-air interface with an $\mathrm{rms}$ height of $2 \mathrm{~m}$. The transmitter and receiver are 17 and $15 \mathrm{~m}$ below the interface and the effective dielectric constant of the canopy is $\varepsilon_{\text {eff }}=1.03+j 0.036$, as before. It is shown that the canopy-air interface roughness increase the dispersion. Fig. 14 shows the impulse response of the forest channel including tree trunks and the underlying ground plane. For the calculation of the impulse response, Gauss quadrature method with 40 points is used over the frequency range of 10-90 MHz. A Gaussian pulse is assumed to be transmitted which is also plotted in Fig. 14 for comparison. The amplitude of the transmitted pulse is multiplied by the path loss and delayed by the free-space distance between the transmitter and receiver. As can be seen in Fig. 14, pulse spreading and ringing are observed which are the result of dispersion and multipath. The last simulation demonstrates performance of different spatial diversity schemes. Three antenna $1.5 \lambda$ apart are aligned on the $x$-axis placed $1 \mathrm{~km}$ away from a transmitter operating at 50 $\mathrm{MHz}$ in the forest. Three diversity schemes are examined: 1) selective diversity (SD); 2) equal gain combining (EG); and 3) maximal ratio (MR) combining [13]. In SD scheme the detector 


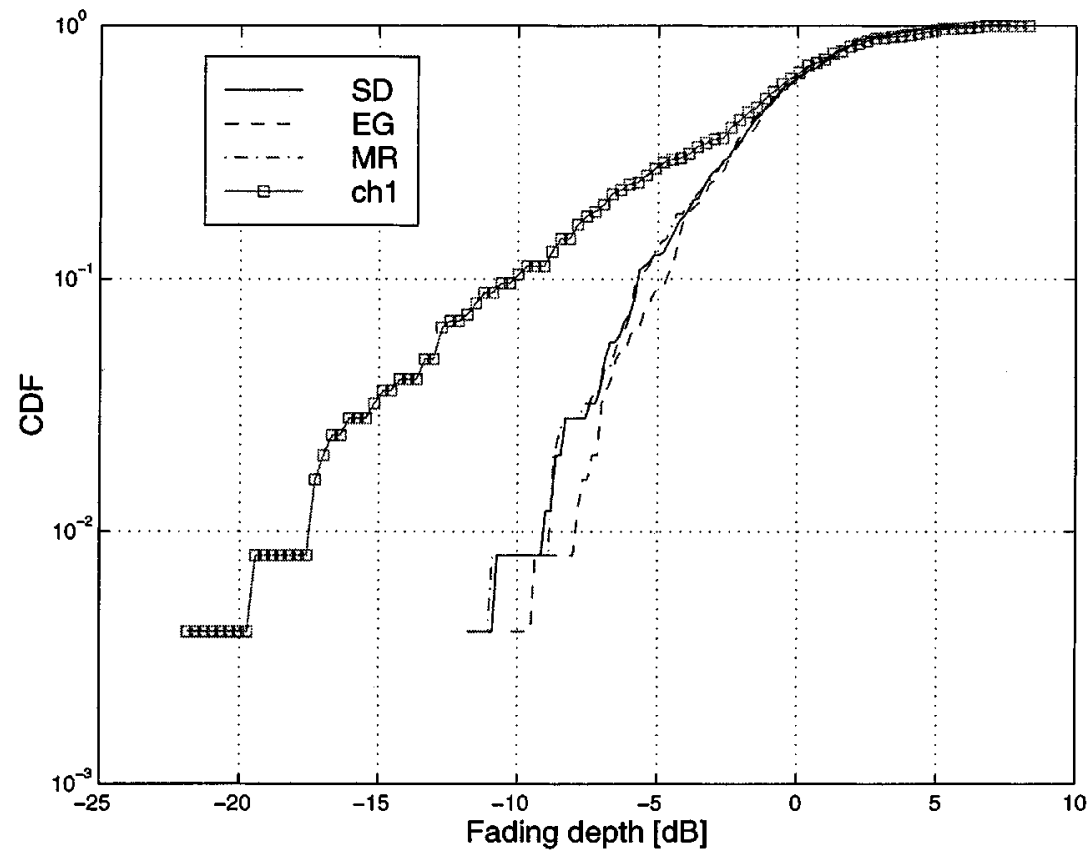

Fig. 15. Cumulative distribution function (CDF) of fading depth for each diversity scheme when antenna arrays are long $x$ axis.

simply chooses the output of the receiver with the highest receiver power. In the EG combining method, the detector is provided with the averaged detected signal from each receiver (a weighting factor of $w_{i}=1 / 3$ is used in the combining). In the diversity scheme based on maximal ratio, combining a weighting factor proportional to the signal power is used to combine the signals from the receiver $\left(w_{i}=\left(P_{i} / \Sigma_{j=1}^{3} P_{j}\right)\right)$. To assess the performance of the above-mentioned diversity combining methods, the cumulative distribution function (CDF) of fading depth for each diversity scheme is calculated using a Monte Carlo simulations. The fading depth is defined as power level at the output of each combiner (SD, EG, or MR) above the average power in $\mathrm{dB}\left(10 \log \left(|\vec{E}|^{2} /\left\langle|\vec{E}|^{2}\right\rangle\right)\right)$. Fig. 15 shows the CDF of fading depth for SD, EG, and MR combining methods. Also shown is the CDF of one of the channels. It is shown that the performance of all three diversity scheme is approximately the same for this problem.

\section{CONCLUSION}

A complete wave propagation model capable of predicting path loss, time-delay response, and coherent frequency response in a forest environment is developed. The model is based on a hybrid analytical and numerical approach which allows for efficient computation of important channel properties without compromising accuracy. The model accounts for multiple scattering among tree trunks and includes the effects of the forest ground plane. Branches and leaves are represented by an effective dielectric constant which limits the range of validity of the model up to UHF. Except for the path-loss calculation, other statistical channel characteristics are determined using a Monte Carlo simulation. In general, propagation simulations are slower at higher frequencies which, depending on the computer platform, also constitutes a limit for the highest frequency. The model is used to simulate performance of antenna arrays and different diver- sity schemes. It is shown that, because of a significant nonzero mean field, spatial diversity only improves the channel fading moderately and there is not a significant difference between different diversity combining schemes. The model presented in this paper shows the possibility of constructing a very accurate physics-based propagation model capable of end-to-end channel simulations.

\section{APPENDIX \\ NEAR-Field CALCULATION}

In this section, an efficient formulation for the calculation of scattered fields from dielectric cylinders is provided. With the help of the Hertz potential, electric field can be easily computed from a known current distribution, $\overrightarrow{\mathbf{J}}$, by using $\overrightarrow{\mathbf{E}}=\nabla\left(\nabla \cdot \vec{\Pi}_{e}\right)+$ $k_{1}^{2} \vec{\Pi}_{e}$. The electrical Hertz vector is represented by

$$
\vec{\Pi}_{e}=-\sum_{n} \frac{1}{4 \pi j w \varepsilon} \int_{v^{\prime}} \vec{J}_{n} e^{-j k_{z}^{\prime} z^{\prime}} \frac{e^{j k\left|\vec{r}-\vec{r}^{\prime}\right|}}{\left|\vec{r}-\vec{r}^{\prime}\right|} d v^{\prime}
$$

where $\vec{J}_{n}$ is a constant vector. Let

$$
\begin{aligned}
I & =\int_{s_{n}^{\prime}} \frac{e^{j k\left|\vec{r}-\vec{r}^{\prime}\right|}}{\left|\vec{r}-\vec{r}^{\prime}\right|} e^{-j k_{z}^{\prime} z^{\prime}} d x^{\prime} d y^{\prime} \\
& =\int_{s_{n}^{\prime}} f\left(\left|\vec{r}-\vec{r}^{\prime}\right|\right) e^{-j k_{z}^{\prime} z^{\prime}} d x^{\prime} d y^{\prime}
\end{aligned}
$$

where $s_{n}^{\prime}$ is a cell area. For small pixel area and using mid-point integration

$$
\int_{s_{n}^{\prime}} \approx \triangle^{2} \frac{e^{j k r_{n}}}{r_{n}}
$$

where $r_{n}=\sqrt{\left(x-x_{n}\right)^{2}+\left(y-y_{n}\right)^{2}+\left(z-z^{\prime}\right)^{2}}$. Based on 
the above result, other integrals can be evaluated as

$$
\begin{aligned}
I_{x x}= & \frac{\partial^{2}}{\partial x^{2}} I \approx \triangle^{2} \frac{e^{j k r_{n}}}{r_{n}}\left(j k r_{n}-1\right) \\
& \cdot\left[\frac{1}{r_{n}}-\frac{3 \cos ^{2} \theta_{n}}{r_{n}}+j k \cos ^{2} \theta_{n}\right] \\
I_{y y}= & \frac{\partial^{2}}{\partial y^{2}} I \approx \triangle^{2} \frac{e^{j k r_{n}}}{r_{n}}\left(j k r_{n}-1\right) \\
& \cdot\left[\frac{1}{r_{n}}-\frac{3 \sin ^{2} \theta_{n}}{r_{n}}+j k \sin ^{2} \theta_{n}\right] \\
I_{z z}= & \frac{\partial^{2}}{\partial z^{2}} I \approx \triangle^{2} \frac{e^{j k r_{n}}}{r_{n}}\left(j k r_{n}-1\right) \\
& \cdot\left[\frac{1}{r_{n}}-\frac{3 \cos ^{2} \theta_{n}}{r_{n}}+j k \cos ^{2} \theta_{n}\right] \\
I_{u}= & \frac{\partial}{\partial u} I \approx \triangle^{2} \frac{j k r_{n}-1}{r_{n}^{2}} e^{j k r_{n}} \begin{cases}\cos \theta_{n} & u=x \\
\sin \theta_{n} & u=y .\end{cases}
\end{aligned}
$$

The rest terms, $I_{x y}, I_{x z}$, and $I_{y z}$, can be evaluated analytically as

$$
\begin{aligned}
I_{x y}= & \triangle^{2}\left[\frac{e^{j k r_{n}^{--}}}{r_{n}^{--}}-\frac{e^{j k r_{n}^{-+}}}{r_{n}^{-+}}-\frac{e^{j k r_{n}^{+-}}}{r_{n}^{+-}}+\frac{e^{j k r_{n}^{++}}}{r_{n}^{++}}\right] \\
\int_{0}^{L} I_{x z} d z^{\prime}= & \frac{\partial^{2}}{\partial x \partial z} \int_{0}^{L} I d z^{\prime} \\
= & \triangle^{2} \frac{e^{j k r_{n 0}}}{r_{n 0}}\left(j k r_{n 0}-1\right) \cos \theta_{n 0} \\
& -\triangle^{2} \frac{e^{j k r_{n l}}}{r_{n 1 l}}\left(j k r_{n l}-1\right) \cos \theta_{n l} e^{-j k_{z}^{\prime} L} \\
\int_{0}^{L} I_{y z} d z^{\prime}= & \frac{\partial^{2}}{\partial y \partial z} \int_{0}^{L} I d z^{\prime} \\
= & \triangle^{2} \frac{e^{j k r_{n 0}}}{r_{n 0}}\left(j k r_{n 0}-1\right) \sin \theta_{n 0} \\
& -\triangle^{2} \frac{e^{j k r_{n l}}}{r_{n l}}\left(j k r_{n l}-1\right) \sin \theta_{n l} e^{-j k_{z}^{\prime} L}
\end{aligned}
$$

where

$$
\begin{aligned}
r_{n}^{ \pm \pm} & \left.=\sqrt{\left(x-x_{n} \pm \triangle / 2\right)^{2}+\left(y-y_{n}\right.} \pm \triangle / 2\right)^{2}+\left(z-z^{\prime}\right)^{2} \\
r_{n 0} & =\sqrt{\left(x-x_{n}\right)^{2}+\left(y-y_{n}\right)^{2}+z^{2}} \\
r_{n l} & =\sqrt{\left(x-x_{n}\right)^{2}+\left(y-y_{n}\right)^{2}+(z-L)^{2}} \\
\cos _{\theta_{n 0}} & =z / r_{n 0}, \quad \cos \theta_{n l}=(z-L) / r_{n 0} .
\end{aligned}
$$

Therefore, the final expression for the electrical field that includes the effect of ground is given by

$$
\begin{aligned}
\overrightarrow{\mathbf{E} \approx} \approx & -\frac{1}{4 \pi j w \varepsilon} \sum_{n} \int_{0}^{L} \\
& \cdot\left[\left\{\left(R_{h}+1\right)\left(I_{x x}+k_{1}^{2} I\right) l_{x}+\left(R_{h}+1\right) I_{x y} l_{y}\right.\right. \\
& \left.+\left(R_{v}+1\right) I_{x z} l_{z}\right\} \hat{x} \\
& +\left\{\left(R_{h}+1\right) I_{x y} l_{x}+\left(R_{h}+1\right)\left(I_{y y}+k_{1}^{2} I\right) l_{y}\right. \\
& \left.+\left(R_{v}+1\right) I_{y z} l_{z}\right\} \hat{y} \\
& +\left\{\left(R_{h}+1\right) I_{x z} l_{x}+\left(R_{h}+1\right) I_{y z} l_{y}\right. \\
& \left.+\left(R_{v}+1\right)\left(I_{z z}+k_{1}^{2} I\right) l_{z}\right\} \\
& \left.+\hat{R}_{h}\left(I_{x z} \hat{x}+I_{y z} \hat{y}+I_{z z} \hat{z}\right)\left(\cos \varphi l_{x}+\sin \varphi l_{y}\right)\right] d z^{\prime}
\end{aligned}
$$

where the summation is over all cells in all cylinders and $R_{h}$, and $R_{v}$ are the ground Fresnel reflection coefficient and also $\hat{R}_{h}=\sin 2 \theta\left(\cos \theta-\sqrt{\kappa-\sin ^{2} \theta} / \kappa \cos \theta+\sqrt{\kappa-\sin ^{2} \theta}\right)[14]$.

\section{REFERENCES}

[1] T. Tamir, "On radio-wave propagation in forest environments," IEEE Trans. Antennas Propagat., vol. AP-15, pp. 806-817, Nov. 1967.

[2] K. Sarabandi and I. Koh, "Effect of canopy-air interface roughness on HF-UHF wave propagation in forest," IEEE Trans. Antennas Propagat., submitted for publication.

[3] F. T. Ulaby, R. K. Moore, and A. K. Fung, Microwave Remote Sensing Active and Passive. Norwood, MA: Artech House, 1982.

[4] K. Sarabandi and P. Siquera, "Numerical scattering analysis for two dimensional dense random media: Characterization of effective permittivity," IEEE Trans. Antennas Propagat., vol. 45, Apr. 1997.

[5] G. F. Carey and J. T. Oden, Finite Elements. Englewood Cliffs, NJ: Prentice-Hall, 1984

[6] F. Harrington, Time-Harmonic Electromagnetic Fields. New York: McGraw-Hill, 1961.

[7] K. Sarabandi, "Electromagnetic scattering from vegetation canopies," Ph.D. thesis, The University of Michigan, 1989.

[8] K. Sarabandi, P. F. Polatin, and F. T. Ulaby, "Monte Carlo simulation of scattering from a layer of vertical cylinder," IEEE Trans. Antennas Propagat., vol. 41, pp. 465-475, Apr. 1993.

[9] W. L. Stutzman and G. A. Thiele, Antenna Theory and Design. New York: Wiley, 1981.

[10] J. G. Proakis, Digital Communications. New York: McGraw-Hill, 1989.

[11] A. Papoulis, Probability, Random Variables, and Stochastic Process. New York: McGraw-Hill, 1984.

[12] W. H. Press, B. P. Flannery, S. A. Teukolsky, and W. T. Vetterling, Numerical Recipes in C. Cambridge: Cambridge University, 1988

[13] W. C. Jakes, Microwave Mobile Communications. New York: IEEE Press, 1994

[14] K. A. Michalski, "On the effective evaluation of integrals arising in the sommerfeld half-space problem," Proc. Inst. Elect. Eng., vol. 132H, pp. 312-318, Aug. 1985.

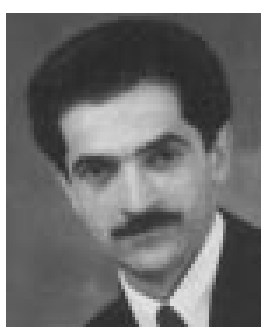

Kamal Sarabandi (S'87-M'90-SM'92-F'00) In 1980, he received the B.S. degree in electrical engineering from Sharif University of Technology, Tehran, Iran. He received the M.S.E. degree in electrical engineering and both the M.S. degree in mathematics and the Ph.D. degree in electrical engineering from University of Michigan in 1986 and 1989 , respectively.

From 1980 to 1984 he worked as a microwave engineer in the Telecommunication Research Center. He is presently an Associate Professor in the Department of Electrical Engineering and Computer Science at the University of Michigan. In the past eight years, he has served as the Principal Investigator or Coinvestigator on many projects sponsored by NASA, JPL, ARO, ONR, ARL, NSF, and numerous industries. He has published many book chapters and more than 85 papers in refereed journals on electromagnetic scattering, random media modeling, wave propagation, microwave measurement techniques, radar calibration, application of neural networks in inverse scattering problems, and microwave sensors. He has also had more than 150 papers and invited presentations in national and international conferences and symposia on similar subjects.

Dr. Sarabandi is a member of the IEEE Geoscience and Remote Sensing ADCOM since January 1998 and served as the Chairman of Geoscience and Remote Sensing Society Southeastern Michigan chapter from 1992 to 1998. He is a member of Commission F of URSI and of the Electromagnetic Academy. He was a recipient of a 1996 Teaching Excellence Award, the 1997 Henry Russel Award from the Regents of the University of Michigan, and the 1999 GAAC Distinguished Lecturer Award from the German Federal Ministry for Education, Science, and Technology. He is listed in American Men and Women of Science and Who's Who in Electromagnetics.

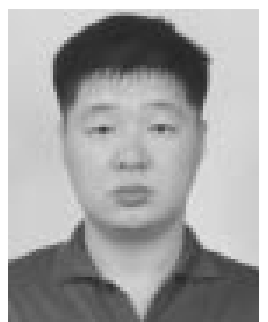

Il-Suek Koh (S'99) was born in Korea. He received the B.S. and M.S. degrees in electronics engineering from Yonsei University, Seoul, Korea, in 1992 and 1994, respectively.

In 1994, he joined LG electronics Ltd. as an Assistant Research Engineer. $\mathrm{He}$ is currently working toward his Ph.D. degree at the University of Michigan, Ann Arbor. His research interests are theoretical modeling of wireless communication channel, multibody problem, and numerical technique for electrically large scatterers. 